\title{
The Key-Dependent Attack on Block Ciphers ${ }^{\star}$
}

\author{
Xiaorui Sun and Xuejia Lai \\ Department of Computer Science \\ Shanghai Jiao Tong University \\ Shanghai, 200240, China \\ sunsirius@sjtu.edu.cn, lai-xj@cs.sjtu.edu.cn
}

\begin{abstract}
In this paper, we formalize an attack scheme using the keydependent property, called key-dependent attack. In this attack, the intermediate value, whose distribution is key-dependent, is considered. The attack determines whether a key is right by conducting statistical hypothesis test of the intermediate value. The time and data complexity of the key-dependent attack is also discussed.

We also apply key-dependent attack on reduced-round IDEA. This attack is based on the key-dependent distribution of certain items in Biryukov-Demirci Equation. The attack on 5.5-round variant of IDEA requires $2^{21}$ chosen plaintexts and $2^{112.1}$ encryptions. The attack on 6 -round variant requires $2^{49}$ chosen plaintexts and $2^{112.1}$ encryptions. Compared with the previous attacks, the key-dependent attacks on 5.5round and 6-round IDEA have the lowest time and data complexity, respectively.
\end{abstract}

Keywords: Block Cipher, Key-Dependent Attack, IDEA.

\section{Introduction}

In current cryptanalysis on block ciphers, widespread attacks use special probability distributions of certain intermediate values. These probability distributions are considered as invariant under different keys used. For example, differential cryptanalysis [7] makes use of the probability of the intermediate differential with high probability. Its value is assumed not to vary remarkably with different keys. Linear cryptanalysis [23] is based on the bias of the linear approximation, which is also generally constant for different keys.

Instead of concentrating on the probability distribution which is invariant for different keys, Ben-Aroya and Biham first proposed the key-dependent property in [2. Key-dependent property means that the probability distribution of intermediate value varies for different keys. In [2], an attack on Lucifer using key-dependent differential was presented. Knudsen and Rijmen also used similar idea to attack DFC in [20].

\footnotetext{
* This work was supported by NSFC Grant No.60573032, 60773092 and 11th PRP of Shanghai Jiao Tong University.
} 
In this paper, we consider the key-dependent property further. The distribution of intermediate value which is key-dependent is called key-dependent distribution. Assume that there are some randomly chosen encryptions. For the intermediate values calculated from these encryptions with the actual key, they should conform to key-dependent distribution. On the other hand, if we use a wrong key to calculate the intermediate values, they are assumed to conform to random distribution. Basing on key-dependent distribution, we formalize a scheme of discovering the actual key by performing statistical hypothesis test [17 on possible keys, and we call this scheme key-dependent attack. For a given key, the null hypothesis of the test is that the intermediate value conforms to the key-dependent distribution determined by the key. The samples of the test are the intermediate values calculated from a few encryptions. If the test is passed, the given key is concluded to be the actual key, otherwise it is discarded. For the keys that share the same key-dependent distribution and the same intermediate value calculation, the corresponding hypothesis tests can be merged to reduce the time needed. By this criterion, the whole key space is divided into several key-dependent subsets.

Due to the scheme of the key-dependent attack, the time complexity of the attack is determined by the time for distinguishing between the random distribution and the key-dependent distribution. The time needed relies on the entropy of the key-dependent distribution: the closer the key-dependent distribution is to the random distribution, the more encryptions are needed. For each key-dependent subset, the number of encryptions and the criteria of rejecting hypothesis can be chosen so that the attack on this subset is optimized. The expected time of the attack on each subset is also obtained.

The total expected time complexity can be calculated from the expected time on each key-dependent subset. Different orders of the key-dependent subsets attacked have different expected time complexities. The order with minimal expected time complexity is presented. The total expected time complexity is also minimized in this way if the actual key is supposed to be chosen uniformly from the whole key space.

This paper also presents a key-dependent attack on block cipher IDEA. The block cipher IDEA (International Data Encryption Algorithm) was proposed in 2122]. The cryptanalysis of IDEA was discussed in

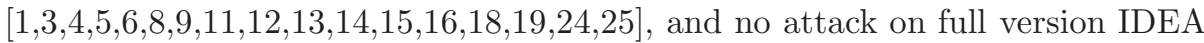
is faster than exhaustive search so far. We investigate the Biryukov-Demirci Equation, which is widely used in recent attacks on IDEA 156131618. We find that particular items of Biryukov-Demirci Equation satisfy key-dependent distribution under some specific constraints. This makes it possible to perform the key-dependent attack on IDEA. Biryukov-Demirci Equation is used to recover the intermediate values from encryptions.

Our key-dependent attack on 5.5-round variant of IDEA requires $2^{21}$ chosen plaintexts and has a time complexity of $2^{112.1}$ encryptions. Our key-dependent attack on the 6 -round variant of IDEA requires $2^{49}$ chosen plaintexts and has a time complexity of $2^{112.1}$ encryptions. These attacks use both fewer chosen 
Table 1. Selected Results of attacks on IDEA

\begin{tabular}{|c|c|c|c|c|}
\hline Rounds & Attack type & Data & Time & Ref. \\
\hline 4.5 & Impossible Differential & $2^{64} \mathrm{CP}$ & $2^{112}$ & 3 \\
\hline 4.5 & Linear & $16 \mathrm{CP}$ & $2^{103}$ & 5 \\
\hline $5^{\dagger}$ & Meet-in-the-Middle & $2^{24} \mathrm{CP}$ & $2^{126}$ & 13 \\
\hline $5^{\dagger}$ & Meet-in-the-Middle & $2^{24.6} \mathrm{CP}$ & $2^{124}$ & 1 \\
\hline 5 & Linear & $2^{18.5} \mathrm{KP}$ & $2^{103}$ & 6 \\
\hline 5 & Linear & $2^{19} \mathrm{KP}$ & $2^{103}$ & 5 \\
\hline 5 & Linear & $16 \mathrm{KP}$ & $2^{114}$ & 6 \\
\hline 5.5 & Higher-Order Differential-Linear & $2^{32} \mathrm{CP}$ & $2^{126.85}$ & 6 \\
\hline 6 & Higher-Order Differential-Linear $2^{6}$ & $64-2^{52} \mathrm{KP}$ & $2^{126.8}$ & 6 \\
\hline $5^{\dagger}$ & Key-Dependent & $2^{17} \mathrm{CP}$ & $2^{125.5}$ & Section 5.3 \\
\hline $5^{\dagger}$ & Key-Dependent & $2^{64} \mathrm{KP}$ & $2^{115.3}$ & Section 5.3 \\
\hline 5.5 & Key-Dependent & $2^{21} \mathrm{CP}$ & $2^{112.1}$ & Section 5.1 \\
\hline 6 & Key-Dependent & $2^{49} \mathrm{CP}$ & $2^{112.1}$ & Section 5.2 \\
\hline
\end{tabular}

CP - Chosen Plaintext, KP - Known Plaintext.

$\dagger$ Attack on IDEA starting from the first round.

plaintexts and less time than all the previous corresponding attacks. We also give two key-dependent attacks on 5-round IDEA starting from the first round. One requires $2^{17}$ chosen plaintexts and needs $2^{125.5}$ encryptions. The other one requires $2^{64}$ known plaintexts and needs $2^{115.3}$ encryptions.We summarize our attacks and previous attacks in Table 1 where the data complexity is measured in the number of plaintexts and the time complexity is measured in the number of encryptions needed in the attack.

The paper is organized as follows: In Section 2 we give a general view of the key-dependent attack. In Section 3 we give a brief description of IDEA block cipher. In Section 4 we show that the probability distribution of some items of the Biryukov-Demirci Equation is a key-dependent distribution. In Section 5 we present two key-dependent attacks on reduced-round IDEA. Section 6 concludes this paper.

\section{The Key-Dependent Attack}

In 2], Ben-Aroya and Biham first proposed the key-dependent property and implemented a key-dependent differential attack on Lucifer. Knudsen and Rijmen also used similar idea to attack DFC in [20.

In this section, we formalize a scheme of identifying the actual key using the following key-dependent property (with high success probability).

Definition 1. For a block cipher, if the probability distribution of an intermediate value varies for different keys under some specific constraints, then this probability distribution is defined as key-dependent distribution. 
Consider some randomly chosen encryptions satisfying the specific constraints. If one uses the actual key to calculate the intermediate value, it should conform to key-dependent distribution. If one uses a wrong key to calculate the intermediate value, it is assumed to be randomly distributed. With such a property, determining whether a given key is right can be done by distinguishing which distribution the intermediate value conforms to, the key-dependent distribution or the random distribution.

We propose an attack scheme, called key-dependent attack, using key-dependent distribution. The attack uses statistical hypothesis test, whose idea is also used in differential and linear attack [17, to distinguish between key-dependent distribution and random distribution. For a key, the null hypothesis of the test is that the intermediate value conforms to the key-dependent distribution determined by the key. Then the attack uses some samples to determine whether the hypothesis is right. The samples of the statistical hypothesis test are the intermediate values obtained from the encryptions satisfying the specific constraints. If the key passes the hypothesis test, the attack concludes that the key is right, otherwise the key is judged to be wrong.

For the keys that share the same key-dependent distribution and the same intermediate value calculation, the corresponding hypothesis tests can be merged. Hence the whole key space is divided into several key-dependent subsets. (Similar idea is proposed in 2.)

Definition 2. A key-dependent subset is a tuple $(P, U)$, where $P$ is a fixed keydependent distribution of intermediate value, and $U$ is a set of keys that share the same key-dependent distribution $P$ and the same intermediate value calculation.

Definition 3. The key fraction $(f)$ of a key-dependent subset is the ratio between the size of $U$ and the size of the whole key space.

The key-dependent attack determines which key-dependent subset the actual key is in by conducting hypothesis tests on each key-dependent subset. Such process on a key-dependent subset $(P, U)$, called individual attack, can be described as the following four phases:

1. Parameter Determining Phase Determine the size of the samples and the criteria of rejecting the hypothesis that the intermediate values conform to $P$.

2. Data Collecting Phase Randomly choose some encryptions according to the specific constraints 1

3. Judgement Phase Calculate the intermediate values from the collected encryptions. If the results satisfy the criteria of rejection, then discard this key-dependent subset, otherwise enter the next phase.

4. Exhaustive Search Phase Exhaustively search $U$ to find the whole key. If the exhaustive search does not find the whole actual key, then start another individual attack on the next key-dependent subset.

${ }^{1}$ Though each individual attack chooses encryptions randomly, one encryption can be used for many individual attacks thus to reduces the total data complexity. 
The time complexity of the key-dependent attack is determined by the time complexity of each individual attack and the order of performing these individual attacks.

For a key-dependent subset $(P, U)$, the time needed for individual attacks relies on the entropy of $P$ : the closer $P$ is to the random distribution, the more difficult the attack is - to ensure the same probability of making the right judgement, the attack needs more encryptions. This indicates that individual attacks for different key-dependent subsets have different time complexities. The time complexity of each individual attack is determined by corresponding key-dependent distribution $P$. For each key-dependent subset, the number of encryptions and the criteria of rejecting hypothesis are then chosen to minimize the time complexity of this individual attack.

To minimize the time complexity of an individual attack, the attack should consider the probability of committing two types of errors: Type I error and Type II error. Type I error occurs when the hypothesis is rejected for a keydependent subset while in fact the actual key is in $U$, and the attack will fail to find the actual key in this case. The probability of Type I error is also defined as significant level, denoted as $\alpha$. Type II error occurs when the test is passed while in fact it is not right, and in this case the attack will come into the exhaustive search phase, but will not find the actual key. The probability of Type II error is denoted as $\beta$. With a fixed size of samples (denoted as $N$ ) and the significance level $\alpha$, the criteria of rejecting the hypothesis is determined, and the probability of Type II error $\beta$ is also fixed. For a fixed size of samples, it is impossible to reduce both $\alpha$ and $\beta$ simultaneously. In order to reduce both $\alpha$ and $\beta$, the attack has to use a larger size of samples, but time and data complexity will increase. Hence, an individual attack needs to balance between the size of samples, and the probability of making wrong judgement.

For a key-dependent subset $(P, U)$, if the actual key is not in this subset, the expected time complexity (measured by the number of encryptions) of the individual attack on this subset is

$$
W=N+\beta|U|
$$

If the actual key is in this subset, the expected time of the individual attack on this subset is

$$
R=N+(1-\alpha) \frac{|U|}{2}
$$

Since the time complexity is dominated by attacking on wrong key-dependent subsets (there is only one key-dependent subset containing the actual key), the attack only needs to minimize the time complexity of the individual attack for each wrong key-dependent subset to minimize the total time complexity. Although $\alpha$ does not appear in Equation (1), $\alpha$ affects the success probability of the attack, so $\alpha$ should also be considered. We set one upper bound of $\alpha$ to ensure that the success probability is above a fixed value, and then choose such size of samples that Equation (1) is minimized, in order to minimize the time complexity of individual attacks. 
In addition, it is entirely possible that some key-dependent distributions is so close to random distribution that the expected time for performing hypothesis tests is longer than directly searching the subsets. For these key-dependent subsets, the attack exhaustively searches the subset directly instead of using statistical hypothesis test method.

On the other hand, the time complexity of the key-dependent attack is also affected by the order of performing individual attacks on different key-dependent subsets. Because the expected time complexities of individual attacks are different, different sequences of performing individual attacks result in different total expected time complexity. Assume that a key-dependent attack performs individual attacks on $m$ key-dependent subsets in the order of $\left(P_{1}, U_{1}\right), \ldots,\left(P_{m}, U_{m}\right)$. Let $R_{i}$ denote the expected time for $\left(P_{i}, U_{i}\right)$ if the actual key is in $U_{i}$, and $W_{i}$ denote the expected time if the actual key is not in $U_{i}$. We have following result:

Theorem 1. The expected time for the whole key-dependent attack is minimal if the following condition is satisfied

$$
\frac{f_{1}}{W_{1}} \geq \frac{f_{2}}{W_{2}} \geq \cdots \geq \frac{f_{m}}{W_{m}}
$$

Proof. The expected time of the attack in the order of $\left(P_{1}, U_{1}\right), \ldots,\left(P_{m}, U_{m}\right)$ is

$$
\begin{aligned}
\Phi= & \left.f_{1}\left[R_{1}+\alpha\left(W_{2}+W_{3}+\cdots+W_{m}\right)\right]+f_{2}\left[W_{1}+R_{2}+\alpha\left(W_{3}+\cdots+W_{m}\right)\right)\right] \\
& +f_{3}\left[W_{1}+W_{2}+R_{3}+\alpha\left(W_{4}+\ldots W_{m}\right)\right]+\cdots+f_{m}\left(W_{1}+W_{2}+\ldots W_{m-1}+R_{m}\right) \\
= & \sum_{i=1}^{m} f_{i} R_{i}+\sum_{i=1}^{m}\left(f_{i} \sum_{j=1}^{i-1} W_{j}\right)+\alpha \sum_{i=1}^{m}\left(f_{i} \sum_{j=i+1}^{m} W_{j}\right)
\end{aligned}
$$

If the attack is performed in the order of $\left(P_{s_{1}}, U_{s_{1}}\right),\left(P_{s_{2}}, U_{s_{2}}\right), \ldots,\left(P_{s_{m}}, U_{s_{m}}\right)$, where $s_{1}, s_{2}, \ldots, s_{m}$ is a permutation of $1,2, \ldots, m$. The expected time is

$$
\Phi^{\prime}=\sum_{i=1}^{m} f_{s_{i}} R_{s_{i}}+\sum_{i=1}^{m}\left(f_{s_{i}} \sum_{j=1}^{i-1} W_{s_{j}}\right)+\alpha \sum_{i=1}^{m}\left(f_{s_{i}} \sum_{j=i+1}^{m} W_{s_{j}}\right)
$$

$f_{i} W_{j}+\alpha f_{j} W_{i}$ occurs in $\Phi$ if and only if $j<i$ and occurs in $\Phi^{\prime}$ if and only if $j^{\prime}<i^{\prime}$ where $s_{i^{\prime}}=i$ and $s_{j^{\prime}}=j$. Hence

$$
\Phi-\Phi^{\prime}=\sum_{j<i \text { and } j^{\prime}>i^{\prime}}\left(f_{i} W_{j}+\alpha f_{j} W_{i}-f_{j} W_{i}-\alpha f_{i} W_{j}\right)
$$

Since $\alpha \leq 1$ and $f_{i} W_{j}-f_{j} W_{i} \leq 0$ for $j<i, \Phi-\Phi^{\prime} \leq 0$ for any permutation $s_{1}, s_{2}, \ldots s_{m}$.

In the following sections of this paper, we present a concrete key-dependent attack on the block cipher IDEA. 


\section{The IDEA Block Cipher}

In this section, we give a brief introduction of IDEA and notations used later in this paper.

IDEA block cipher encrypts a 64 -bit plaintext with a 128 -bit key by an 8.5 round encryption. The fifty-two 16-bit subkeys are generated from the 128bit key $Z$ by key-schedule algorithm. The subkeys are generated in the order $Z_{1}^{1}, Z_{2}^{1}, \ldots, Z_{6}^{1}, Z_{1}^{2}, \ldots, Z_{6}^{8}, Z_{1}^{9}, \ldots, Z_{4}^{9}$. The key $Z$ is partitioned into eight 16 -bit words which are used as the first eight subkeys. The key $Z$ is then cyclically shifted to the left by 25 bits, and then generate the following eight subkeys. This process is repeated until all the subkeys are obtained. In Table 2 , the correspondence between the subkeys and the key $Z$ is directly given.

The block cipher partitions the 64-bit plaintext into four 16-bit words and uses three different group operations on pairs of 16-bit words: exclusive OR, denoted by $\oplus$; modular addition $2^{16}$, denoted by $\boxplus$ and modular multiplication $2^{16}+1\left(0\right.$ is treated as $\left.2^{16}\right)$, denoted by $\odot$.

As Figure1, each round of IDEA contains three layers: KA layer, MA layer and Permutation layer. We denote the 64-bit input of round $i$ by $X^{i}=\left(X_{1}^{i}, X_{2}^{i}, X_{3}^{i}, X_{4}^{i}\right)$. In the KA layer, the first and the fourth words are modular multiplied with $Z_{1}^{i}$ and $Z_{4}^{i}$ respectively. The second and the third words are modular added with $Z_{2}^{i}$ and $Z_{3}^{i}$ respectively. The output of the KA layer is denoted by $Y^{i}=\left(Y_{1}^{i}, Y_{2}^{i}, Y_{3}^{i}, Y_{4}^{i}\right)$.

In the MA layer, two intermediate values $p^{i}=Y_{1}^{i} \oplus Y_{3}^{i}$ and $q^{i}=Y_{2}^{i} \oplus Y_{4}^{i}$ are computed first. These two values are processed to give $u^{i}$ and $t^{i}$,

$$
\begin{gathered}
u^{i}=\left(p^{i} \odot Z_{5}^{i}\right) \boxplus t^{i} \\
t^{i}=\left(\left(p^{i} \odot Z_{5}^{i}\right) \boxplus q^{i}\right) \odot Z_{6}^{i}
\end{gathered}
$$

We denote $s^{i}$ the intermediate value $p^{i} \oplus Z_{5}^{i}$ for convenience. The output of the MA layer is then permutated to give the output of this round $\left(Y_{1}^{i} \oplus u^{i}, Y_{3}^{i} \oplus\right.$ $\left.u^{i}, Y_{2}^{i} \oplus t^{i}, Y_{4}^{i} \oplus t^{i}\right)$, which is also the input of round $i+1$, denoted by $\left(X_{1}^{i+1}, X_{2}^{i+1}\right.$, $\left.X_{3}^{i+1}, X_{4}^{i+1}\right)$. The complete diffusion, which means every bit of $\left(X_{1}^{i+1}, X_{2}^{i+1}, X_{3}^{i+1}\right.$, $\left.X_{4}^{i+1}\right)$ is affected by every bit of $\left(Y_{1}^{i}, Y_{2}^{i}, Y_{3}^{i}, Y_{4}^{i}\right)$, is obtained in the MA layer.

Table 2. The Key-Schedule of IDEA

\begin{tabular}{|c|cccccc|}
\hline Round & $Z_{1}^{2}$ & $Z_{2}^{2}$ & $Z_{3}^{2}$ & $Z_{4}^{2}$ & $Z_{5}^{2}$ & $Z_{6}^{2}$ \\
\hline 1 & $0-15$ & $16-31$ & $32-47$ & $48-63$ & $64-79$ & $80-95$ \\
2 & $96-111$ & $112-127$ & $25-40$ & $41-56$ & $57-72$ & $73-88$ \\
3 & $89-104$ & $105-120$ & $121-8$ & $9-24$ & $50-65$ & $66-81$ \\
4 & $82-97$ & $98-113$ & $114-1$ & $2-17$ & $18-33$ & $34-49$ \\
5 & $75-90$ & $91-106$ & $107-122$ & $123-10$ & $11-26$ & $27-42$ \\
6 & $43-58$ & $59-74$ & $100-115$ & $116-3$ & $4-19$ & $20-35$ \\
7 & $36-51$ & $52-67$ & $68-83$ & $84-99$ & $125-12$ & $13-28$ \\
8 & $29-44$ & $45-60$ & $61-76$ & $77-92$ & $93-108$ & $109-124$ \\
9 & $22-37$ & $38-53$ & $54-69$ & $70-85$ & & \\
\hline
\end{tabular}




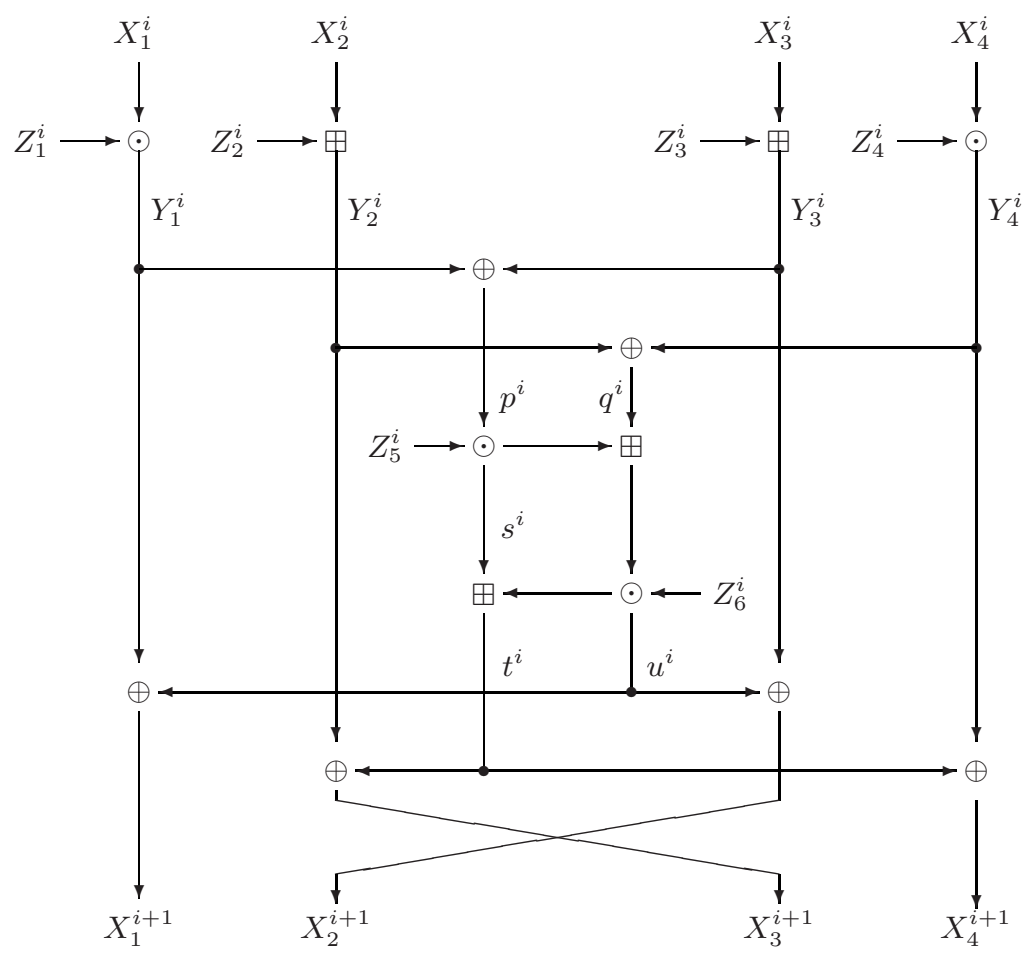

Fig. 1. Round $i$ of IDEA

In this paper, we will use $P=\left(P_{1}, P_{2}, P_{3}, P_{4}\right)$ and $P^{\prime}=\left(P_{1}^{\prime}, P_{2}^{\prime}, P_{3}^{\prime}, P_{4}^{\prime}\right)$ to denote a pair of plaintexts, where $P_{i}$ and $P_{i}^{\prime}$ are 16 -bit words. $C=\left(C_{1}, C_{2}, C_{3}, C_{4}\right)$ and $C^{\prime}=\left(C_{1}^{\prime}, C_{2}^{\prime}, C_{3}^{\prime}, C_{4}^{\prime}\right)$ are their ciphertexts respectively. We also use the symbol ' to distinguish the intermediate values corresponding to $P^{\prime}$ from to $P$. For example, $s^{i}$ is obtained from plaintext $P$ and $P^{\prime}$ will generate $s^{\prime}$. The notation $\Delta$ will denote the XOR difference, for instance, $\Delta s^{i}$ is equal to $s^{i} \oplus s^{i}$.

\section{The Key-Dependent Distribution of IDEA}

In this section, we describe the key-dependent distribution of the block cipher IDEA, which will be used in our attack later. The notations used are the same as in 6].

The Biryukov-Demirci relation was first proposed by Biryukov [16 and Demirci 13. Many papers have discussed attacking on IDEA using this relation, such as 156131618 . The relation can be written in following form ( $L S B$ denotes the least significant bit) 


$$
\begin{aligned}
L S B\left(C_{2} \oplus C_{3}\right)= & L S B\left(P_{2} \oplus P_{3} \oplus Z_{2}^{1} \oplus Z_{3}^{1} \oplus s^{1} \oplus Z_{2}^{2} \oplus Z_{3}^{2} \oplus s^{2}\right. \\
& \oplus Z_{2}^{3} \oplus Z_{3}^{3} \oplus s^{3} \oplus Z_{2}^{4} \oplus Z_{3}^{4} \oplus s^{4} \oplus Z_{2}^{5} \oplus Z_{3}^{5} \oplus s^{5} \\
& \oplus Z_{2}^{6} \oplus Z_{3}^{6} \oplus s^{6} \oplus Z_{2}^{7} \oplus Z_{3}^{7} \oplus s^{7} \oplus Z_{2}^{8} \oplus Z_{3}^{8} \oplus s^{8} \\
& \left.\oplus Z_{2}^{9} \oplus Z_{3}^{9}\right)
\end{aligned}
$$

It is shown in [5] that, for two pairs of plaintext and ciphertext $(P, C)$ and $\left(P^{\prime}, C^{\prime}\right)$, XOR their corresponding Biryukov-Demirci relation, we will obtain from Equation (3)

$$
\begin{aligned}
L S B\left(C_{2} \oplus C_{3} \oplus C_{2}^{\prime} \oplus C_{3}^{\prime}\right)= & L S B\left(P_{2} \oplus P_{3} \oplus P_{2}^{\prime} \oplus P_{3}^{\prime} \oplus \Delta s^{1} \oplus \Delta s^{2}\right. \\
& \left.\oplus \Delta s^{3} \oplus \Delta s^{4} \oplus \Delta s^{5} \oplus \Delta s^{6} \oplus \Delta s^{7} \oplus \Delta s^{8}\right)
\end{aligned}
$$

We call Equation (4) Biryukov-Demirci Equation.

The following theorem shows that the probability distribution of $\operatorname{LSB}\left(\Delta s^{i}\right)$ in Biryukov-Demirci Equation is a key-dependent distribution.

Theorem 2. Consider round $i$ of IDEA. If one pair of intermediate value $\left(p^{i}, p^{i}\right)$ satisfies $\Delta p^{i}=8000_{x}$, then the probability of $\operatorname{LSB}\left(\Delta s^{i}\right)=\operatorname{LSB}\left(8000_{x} \odot Z_{5}^{i}\right)$ is

$$
\operatorname{Prob}\left(L S B\left(\Delta s^{i}\right)=L S B\left(8000_{x} \odot Z_{5}^{i}\right)\right)=\frac{\# W}{2^{15}}
$$

where $W$ is the set of all such 16-bit words $w$ that $1 \leq w \leq 8000_{x}$ and that

$$
\left(w * Z_{5}^{i}\right)+\left(8000_{x} * Z_{5}^{i}\right)<2^{16}+1
$$

where * is defined as

$$
a * b= \begin{cases}a \odot b & \text { if } a \odot b \neq 0 \\ 2^{16} \quad \text { if } a \odot b=0\end{cases}
$$

Proof. Consider every intermediate pair $\left(p^{i}, p^{\prime i}\right)$ which satisfies $\Delta p^{i}=8000_{x}$, excluding $\left(0,8000_{x}\right)$. We have $p^{i}=p^{i}+8000_{x}$ or $p^{i}=p^{i}+8000_{x}$. Without losing generality, assume $p^{i}=p^{i}+8000_{x}$, where $1 \leq p^{i}<8000_{x}$ and $8000_{x}<p^{i}<2^{16}$.

If we consider only the least significant bit, $\operatorname{LSB}\left(s^{i}\right)=L S B\left(p^{i} * Z_{5}^{i}\right)$. The following equations also hold

$$
\begin{aligned}
\operatorname{LSB}\left(s^{i}\right) & =L S B\left(p^{i} \odot Z_{5}^{i}\right) \\
& =L S B\left(p^{\prime i} * Z_{5}^{i}\right) \\
& =L S B\left(\left(p^{i}+8000_{x}\right) * Z_{5}^{i}\right) \\
& =L S B\left(\left(\left(p^{i} * Z_{5}^{i}\right)+\left(8000_{x} * Z_{5}^{i}\right)\right) \quad\left(\bmod 2^{16}+1\right)\right)
\end{aligned}
$$

In the special case when $\left(p^{i}, p^{i}\right)$ is $\left(0,8000_{x}\right)$, let $p^{i}=8000_{x}$, and $p^{i}=0$. The Equations (6) also holds, because $p^{\prime i}=0$ is actually treated as $2^{16}$ for inputs of $\odot$ and $*$. 


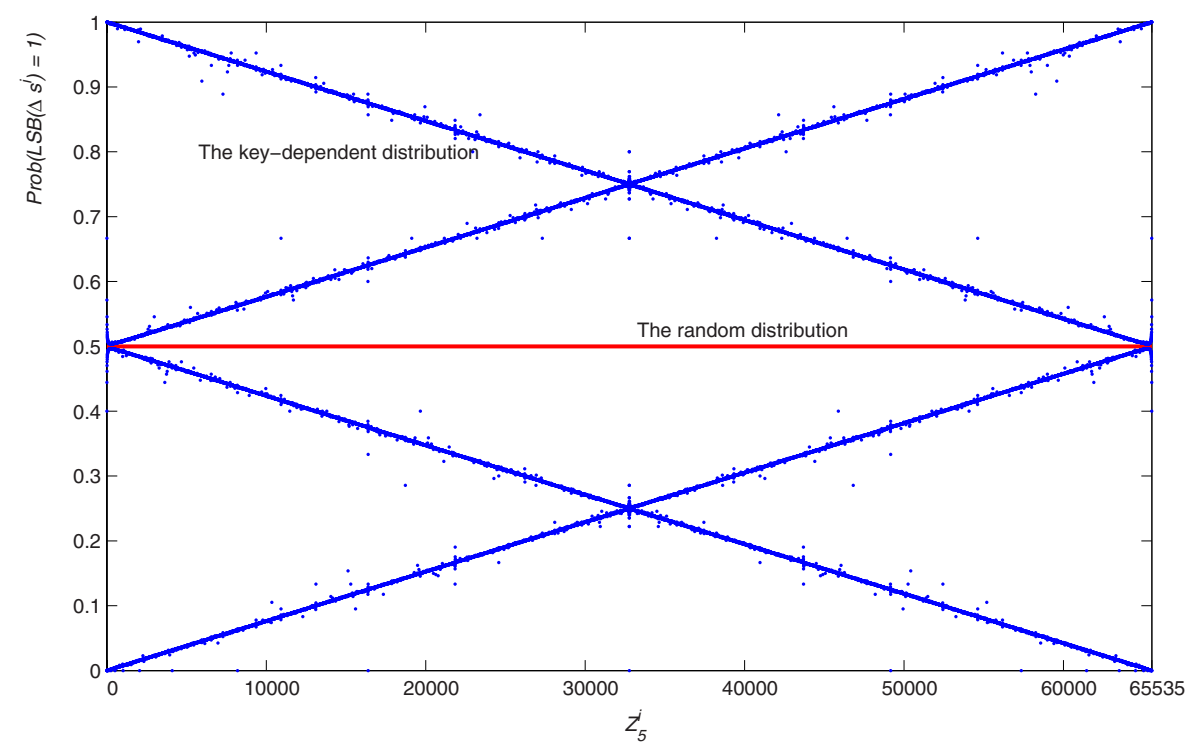

Fig. 2. The key-dependent distribution of $\operatorname{Prob}(L S B(\Delta s)=1)$ on the value of $Z_{5}^{i}$

If $\left(p^{i} * Z_{5}^{i}\right)+\left(8000_{x} * Z_{5}^{i}\right)$ is smaller than $2^{16}+1$, then $L S B\left(s^{i}\right)=L S B\left(s^{i}\right) \oplus$ $L S B\left(8000_{x} * Z_{5}^{i}\right)$ holds because of the equivalence of XOR and modular addition for the least significant bit. Moreover, $\operatorname{LSB}\left(\Delta s^{i}\right)=L S B\left(8000_{x} * Z_{5}^{i}\right)$ is satisfied, which means $\operatorname{LSB}\left(\Delta s^{i}\right)=\operatorname{LSB}\left(8000_{x} \odot Z_{5}^{i}\right)$

Otherwise, $L S B\left(s^{i}\right)$ is equal to $L S B\left(s^{i}\right) \oplus L S B\left(8000_{x} * Z_{5}^{i}\right) \oplus 1$ because of the carry. So $\operatorname{LSB}\left(\Delta s^{i}\right)$ equals to $\operatorname{LSB}\left(8000_{x} \odot Z_{5}^{i}\right) \oplus 1$.

Therefore, we may conclude that $\operatorname{LSB}\left(\Delta s^{i}\right)=L S B\left(8000_{x} \odot Z_{5}^{i}\right)$ if and only if the pair $\left(p^{i}, p^{i}\right)$ satisfies $\left(w * Z_{5}^{i}\right)+\left(8000_{x} * Z_{5}^{i}\right)<2^{16}+1$, where $w$ is either $p^{i}$ or $p^{i}$, whichever between 1 and $8000_{x}$. And there are at most $2^{15}$ such $w$, hence Equation (6) holds. This completes the proof.

Remark 1. Figure 2 plots the relation between the subkey $Z_{5}^{i}$ and the probability of $L S B\left(\Delta s^{i}\right)=1$. As shown in Figure 2 for most $Z_{5}^{i}$, the probability of $\operatorname{LSB}\left(\Delta s^{i}\right)=1$ is different from random distribution. Hence, it is possible to perform key-dependent attack on IDEA using this key-dependent distribution.

For most $Z_{5}^{i}$, there are general four cases for the probability of $\operatorname{LSB}\left(\Delta s^{i}\right)=1$ as $Z_{5}^{i}$ grows from 0 to $2^{16}-1$, which can be roughly approximated as following:

$$
\operatorname{Prob}\left(L S B\left(\Delta s^{i}\right)=1\right) \approx \begin{cases}\frac{Z_{5}^{i}}{2^{17}} & \text { last two bits of } Z_{5}^{i}=00 \\ 0.5-\frac{Z_{5}^{i}}{2^{17}} & \text { last two bits of } Z_{5}^{i}=01 \\ 1.0-\frac{Z_{5}^{i}}{2^{17}} & \text { last two bits of } Z_{5}^{i}=10 \\ 0.5+\frac{Z_{5}^{i}}{2^{17}} & \text { last two bits of } Z_{5}^{i}=11\end{cases}
$$


From Equation (7), following approximation also holds for most $Z_{5}^{i}$

$\min \left\{\operatorname{Prob}\left(L S B\left(\Delta s^{i}\right)=0\right), \operatorname{Prob}\left(L S B\left(\Delta s^{i}\right)=1\right)\right\} \approx \begin{cases}\frac{Z_{5}^{i}}{2^{17}}, & \operatorname{LSB}\left(Z_{5}^{i}\right)=0 \\ 0.5-\frac{Z_{5}^{i}}{2^{17}}, & \operatorname{LSB}\left(Z_{5}^{i}\right)=1\end{cases}$

Calculation shows that, for only 219 out of all $2^{16}$ possible $Z_{5}^{i}$, the difference between the approximation (Equation (7) or (8)) and the accurate provability is larger than 0.01 .

Equation (8) indicates that we can approximate left hand side of Equation (8) by fixing several most significant bits and the least significant bit. In following sections, we will show that we only need to distinguish the approximate probability distribution from random distribution. Hence, for most $Z_{5}^{i}$, this approximation is close enough to the accurate value. For $Z_{5}^{i}$ that can not be approximated in this way, we use other methods to deal with this situation.

\section{The Key-Dependent Attack on IDEA}

In this section, we will present two key-dependent attacks on reduced-round IDEA. In Section 5.1, we will give a basic attack on the 5.5-round variant of IDEA and then extend it to 6-round variant in Section 5.2. We also give two keydependent attacks on 5-round IDEA starting from the first round in Section 5.3.

\subsection{The Attack on 5.5-Round Variant of IDEA}

We first present one key-dependent attack on the 5.5-round variant of IDEA. The attack starts from the third round and ends before the MA layer of the eighth round. The main idea of this attack is to perform key-dependent attack based on the key-dependent distribution of $\Delta s^{4}$ described in Theorem 2.

Consider the 5.5-round variant of IDEA starting from the third round, the Biryukov-Demirci Equation can be rewritten as

$$
L S B\left(\Delta s^{4}\right)=L S B\left(P_{2} \oplus P_{3} \oplus P_{2}^{\prime} \oplus P_{3}^{\prime} \oplus C_{2} \oplus C_{3} \oplus C_{2}^{\prime} \oplus C_{3}^{\prime} \oplus \Delta s^{3} \oplus \Delta s^{5} \oplus \Delta s^{6} \oplus \Delta s^{7}\right)
$$

Where $P$ and $P^{\prime}$ are equivalent to $X^{3}$ and $X^{\prime 3}, C$ and $C^{\prime}$ are equivalent to $Y^{8}$ and $Y^{\prime 8}$ by the variant of IDEA.

We first construct a pair of plaintexts satisfying the specific constraint $\Delta p^{4}=$ $8000 x$. The construction is based on the following lemma.

Lemma 1. For any $\alpha$, if two 16-bit words $x$ and $x^{\prime}$ have the same least 15 significant bits, then

- $x \oplus \alpha$ and $x^{\prime} \oplus \alpha$ have the same least 15 significant bits,

- $x \boxplus \alpha$ and $x^{\prime} \boxplus \alpha$ have the same least 15 significant bits.

Based on Lemma 1, the following proposition can be obtained. 
Proposition 1. If a pair of intermediate values $Y^{3}$ and $Y^{\prime 3}$ satisfy the following conditions:
a. $\Delta Y_{1}^{3}=\Delta Y_{3}^{3}=0$
b. $\Delta Y_{2}^{3}=8000_{x}$
c. $Y_{2}^{3} \oplus Y_{4}^{3}=Y_{2}^{\prime 3} \oplus Y_{4}^{\prime 3}$

then $\Delta s^{3}=0$ and the probability of $\operatorname{LSB}\left(\Delta s^{4}\right)=0$ can be determined by Equation (5).

Proof. From Condition (a), $\Delta Y_{1}^{3}=\Delta Y_{3}^{3}=0, p^{3}$ is equal to $p^{\prime 3}$. Then $\Delta s^{3}=0$ is quite straightforward.

From Condition (c), $q^{3}$ is equal to $q^{\prime 3}$. If $p^{3}$ and $q^{3}$ are fixed, $u^{3}$ and $t^{3}$ are also fixed with respect to any $Z_{5}^{3}$ and $Z_{6}^{3}$. It indicates that $X_{1}^{4}=Y_{1}^{3} \oplus u^{3}=X_{1}^{\prime 4}$. Note that $Y_{1}^{4}$ and $Y_{1}^{\prime 4}$ are the results of modular-multiplying $X_{1}^{4}$ and $X_{1}^{\prime 4}$ with the same $Z_{1}^{4}$, hence $Y_{1}^{4}$ is equal to $Y_{1}^{\prime 4}$.

On the other hand, $\Delta Y_{2}^{3}=8000_{x}$ means that the least significant 15 bits of $Y_{2}^{3}$ are equal to those of $Y_{2}^{\prime 3}$ and the most significant bit of $Y_{2}^{3}$ and that of $Y_{2}^{\prime 3}$ are different. Because $u^{3}$ is fixed, by Lemma 1, the least significant 15 bits of $X_{3}^{4}$ are equal to those of $X_{3}^{\prime 4}$. Then $\Delta X_{3}^{4}$ is equal to $8000_{x}$ and $\Delta Y_{3}^{4}=8000_{x}$ is obtained by modular addition with the same $Z_{3}^{4}$. From $\Delta Y_{1}^{4}=0$ and $\Delta Y_{3}^{4}=8000_{x}, \Delta p^{4}$ is $8000_{x}$. By Theorem 2 the conclusion is obtained.

In our attack, we use the plaintext pairs satisfying Proposition 1 We obtain Condition (a) by letting $\Delta P_{1}=\Delta P_{3}=0$. By Lemma 2, $P_{2}$ and $P_{2}^{\prime}$ are fixed to have the same least significant 15 bits, and hence $\Delta Y_{2}^{1}=8000_{x}$. In order to fulfill Condition (c), we have to guess $Z_{4}^{3}$ and then according to this guess, to choose $P_{4}$ and $P_{4}^{\prime}$ which satisfy $\Delta Y_{4}^{3}=8000_{x}$.

By Proposition 11 $\Delta s^{3}$ is equal to zero. In order to get the right hand side of Equation (9), we still need to get $\Delta s^{5}, \Delta s^{6}, \Delta s^{7}$. We need to guess $Z_{5}^{5}, Z_{1}^{6}$, $Z_{2}^{6}, Z_{5}^{6}, Z_{6}^{6}, Z_{1}^{7}, Z_{2}^{7}, Z_{3}^{7}, Z_{4}^{7} Z_{5}^{7}, Z_{6}^{7}, Z_{1}^{8}, Z_{2}^{8}, Z_{3}^{8}, Z_{4}^{8}$. As shown in [6], one can partially decrypt one pair of encryptions using these 15 subkeys to calculate the values of $\Delta s^{5}, \Delta s^{6}, \Delta s^{7}$. These 15 subkeys only take key bits $125-99$ and also cover the subkey $Z_{4}^{3}$. Hence, for one guessed 103 key bits, we can calculate the value of $\Delta s^{4}$ from a special pair of encryptions.

We also note that these 103 bits also cover the key $Z_{5}^{4}$, which determine the key-dependent distribution on $\Delta s^{4}$ according to Theorem 2 Therefore, we can perform the key-dependent attack on 5.5-round variant of IDEA. As described in Section 2, the key space can be divided into $2^{103}$ key-dependent subsets by the 103 key bits, each contains $2^{25}$ keys.

For a key-dependent subset $(P, U)$, let $p$ denote the probability of $\operatorname{LSB}\left(\Delta s^{4}\right)=$ $L S B\left(8000_{x} \odot Z_{5}^{4}\right)$. For simplicity, in the following analysis, we assume that $p \leq$ 0.5 , the case when $p>0.5$ is similar. Assume the size of the samples is $n$ pairs of encryptions that satisfy the specific constraint on this key-dependent subset, and $t$ of them satisfy $\operatorname{LSB}\left(\Delta s^{4}\right)=L S B\left(8000_{x} \odot Z_{5}^{4}\right)$. The criteria for not rejecting the hypothesis is that $t$ is smaller or equal to a fixed value $k$. The probability of 
Type I error is

$$
\alpha=\sum_{i=k+1}^{n}\left(\begin{array}{l}
n \\
i
\end{array}\right) p^{i}(1-p)^{n-i}
$$

Type II error is

$$
\beta=\sum_{i=0}^{k}\left(\begin{array}{l}
n \\
i
\end{array}\right) 0.5^{n}
$$

If $(P, U)$ is a wrong key-dependent subset, the expected time complexity of checking this subset is

$$
W=2 n+2^{25} \beta
$$

As shown in Section 2, the attack sets $\alpha$ smaller than or equal to 0.01 to ensure that the probability of the false rejection will not exceed 0.01 . Under this precondition, the attack chooses $n$ and $\beta$ so that $\alpha<0.01$ and minimizes Equation (10) to minimize the time complexity on each key-dependent subset $(P, U)$. By Section 2 we minimize the total expected time complexity with this method. Because this choice is related only to the key $Z_{5}^{4}$, so we only need to get $n$ and $k$ for $2^{16}$ different values.

For example, for a key-dependent subset $(P, U)$ with $Z_{5}^{4}=8001_{x}, p$ is about 0.666687. The attack checks every possible $n$ and $k$ to find the minimized expected time complexity of the individual attack for this subset. As shown in Section 2, the expected time complexity for each subset is upper bounded by exhaustive search on the subset, which is $2^{25}$ in this attack. Hence, the attack only

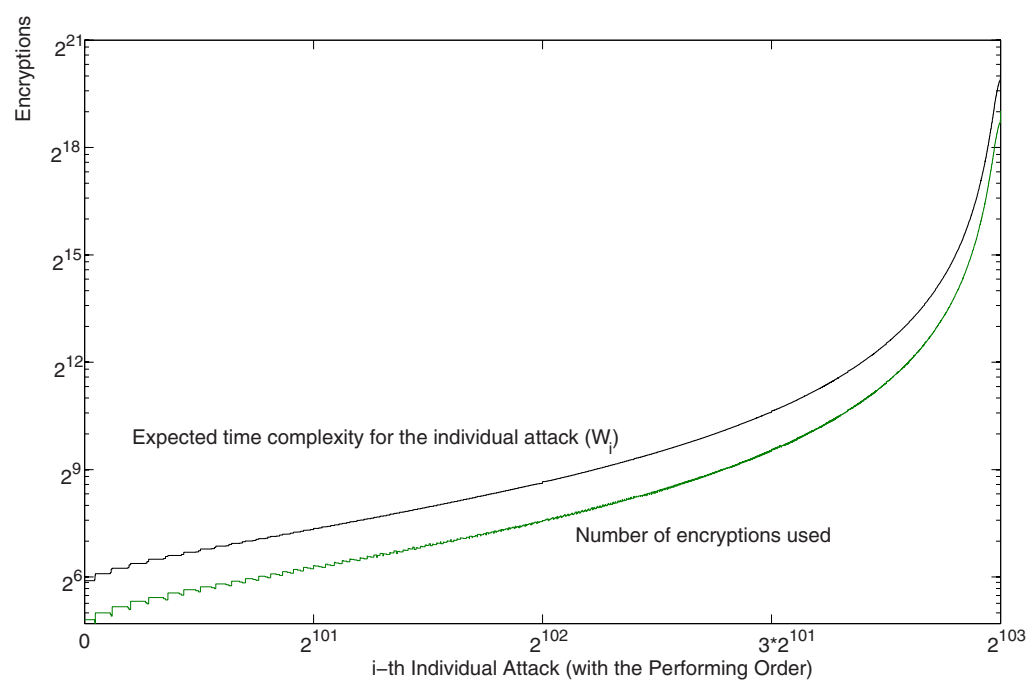

Fig. 3. The number of encryptions used and expected time complexity for individual attacks 
checks all the $n$ and $k$ smaller than $2^{25}$. The expected time is minimized with precondition $\alpha<0.01$ when $n=425$ and $k=164$. In this case, $\alpha=0.009970$, $\beta=0.000001$ and $W=899.094678$.

Since all the key-dependent subsets have the same key fraction, the order of performing individual attacks with minimal expected time complexity becomes the ascending order of $W$ for all key-dependent subsets due to Theorem 1 Figure 3 plots the number of encryptions used and expected time complexity for all the individual attacks.

The total expected time complexity of the attack, described as Equation (2), becomes

$$
\begin{aligned}
\Phi & =\sum_{i=1}^{m} f_{i} R_{i}+\sum_{i=1}^{m}\left(f_{i} \sum_{j=1}^{i-1} W_{j}\right)+\alpha \sum_{i=1}^{m}\left(f_{i} \sum_{j=i+1}^{m} W_{j}\right) \\
& =\frac{1}{2^{103}}\left(\sum_{i=1}^{2^{103}} R_{i}+\sum_{i=1}^{2^{103}} \sum_{j=1}^{i-1} W_{j}+0.01 \sum_{i=1}^{2^{103}} \sum_{j=i+1}^{2^{103}} W_{j}\right) \\
& \leq \frac{1}{2^{103}}\left(\sum_{i=1}^{2^{103}} 2^{26}+\sum_{i=1}^{2^{103}} \sum_{j=1}^{i-1} W_{j}+0.01 \sum_{i=1}^{2^{103}} \sum_{j=i+1}^{2^{103}} W_{j}\right) \\
& =\frac{1}{2^{103}}\left(2^{103} \cdot 2^{26}+\sum_{i=1}^{2^{103}}\left(2^{103}-i+0.01 i\right) W_{i}\right) \\
& \approx 2^{112.1}
\end{aligned}
$$

with $99 \%$ success probability if the attack chooses $n$ and $\beta$ for each key-dependent set and determines the order of performing individual attacks as shown above. The number of pairs needed in one test is about $2^{19}$ in the worst case. The attack uses a set of $2^{21}$ plaintexts, which can provide $2^{20}$ plaintext pairs satisfying the conditions in Proposition 1 for each key-dependent subset.

The attack is summarized as follows:

1. For every possible $Z_{5}^{4}$, calculate the corresponding number of plaintext pairs needed $n$ and the criteria of not rejecting the hypothesis $k$.

2. Suppose $S$ is an empty set. Randomly enumerate a 16-bit word $s$, insert $s$ and $s \oplus 8000_{x}$ into the set $S$. Repeat this enumeration until set $S$ contains $2^{5}$ different words. Ask for the encryption of all the plaintexts of the form $(A, B, C, D)$, where $A$ and $C$ are fixed to two arbitrary constants, $B$ takes all the values in $S$ and $D$ takes all the 16-bit possible values.

3. Enumerate the key-dependent sets in ascending order of $W$ :
(a) Randomly choose a set of plaintext pairs with cardinality $n$ from the known encryptions. The plaintext pairs must satisfy the requirements of Proposition 1
(b) Partially decrypt all the selected encryption pairs and count the occur- rence of $L S B\left(\Delta s_{4}\right)=1$.
(c) Test the hypothesis. If the hypothesis is not rejected, perform exhaustive search for the remaining 25 key bits.




\subsection{The Attack on 6-Round Variant of IDEA}

We now extend the 5.5-round attack to an attack on the 6-round variant of IDEA starting before the MA layer of the second round. The data complexity of the attack is $2^{49}$ and the time complexity is $2^{112.1}$.

As shown in [6], $Z_{5}^{2}$ and $Z_{6}^{2}$ are included in the 103 key bits in the 5.5round attack. Hence, we can add this half round to the 5.5-round attack without enlarging the time complexity.

It is more difficult to construct right plaintext pairs satisfying Proposition 1 Consider a pair of intermediate values $X^{3}$ and $X^{\prime 3}$ before the third round, which satisfy Proposition 1 . If we partially decrypt $X^{3}$ and $X^{\prime 3}$ using any possible $Z_{5}^{2}$ and $Z_{6}^{2}$, the only fact we know is that all the results have the same XOR of the first and third words. The attack hence selects all the plaintexts $P$ where the least 15 significant bits of $P_{1} \oplus P_{3}$ are fixed to an arbitrary 15-bit constant. The total number of selected plaintexts is $2^{49}$. It is possible to provide $2^{48}$ plaintext pairs satisfying the conditions in Proposition 1 in the test for any $Z_{5}^{2}, Z_{6}^{2}$ and $Z_{4}^{3}$. This number is sufficient in any situation.

\subsection{Two Key-Dependent Attacks on 5-Round IDEA Starting from the First Round}

We apply the key-dependent attack to the 5-round IDEA starting from the first round. Biryukov-Demirci Equation is reduced to

$$
\begin{aligned}
& L S B\left(\Delta s^{2}\right)=L S B\left(P_{2} \oplus P_{3} \oplus P_{2}^{\prime} \oplus P_{3}^{\prime} \oplus C_{2}\right. \\
& \left.\oplus C_{3} \oplus C_{2}^{\prime} \oplus C_{3}^{\prime} \oplus \Delta s^{1} \oplus \Delta s^{3} \oplus \Delta s^{4} \oplus \Delta s^{5}\right)
\end{aligned}
$$

We choose the plaintext pairs to satisfy Proposition 1 before the first round by guessing $Z_{4}^{1}$, and then $\Delta s^{1}$ is equal to 0 as shown in Section 5.1. In order to determine the right hand side of Equation (11), we need to know $Z_{5}^{3}, Z_{1}^{4}$, $Z_{2}^{4}, Z_{5}^{4}, Z_{6}^{4}, Z_{1}^{5}, Z_{2}^{5}, Z_{3}^{5}, Z_{4}^{5}, Z_{5}^{5}, Z_{6}^{5}$. These 12 subkeys take the bits $75-65$ from key $Z$. These 119 bits only cover the most significant nine bits of $Z_{5}^{2}$, which determines the probability distribution of $L S B\left(\Delta s^{2}\right)$. It is not necessary to guess the complete subkey $Z_{5}^{2}$. The attack continues to guess the least significant bit of $Z_{5}^{2}$ (the 72 nd bit of $Z$ ), and estimates the probability of $\operatorname{LSB}\left(\Delta s^{2}\right)=1$ by Remark 1 instead. Hence, the attack divides the key space into $2^{120}$ keydependent subsets by the 120 key bits, and performs the individual attacks on each key-dependent subset. The attack uses statistical hypothesis test method to determine which subset the actual key is in. For the subkeys $Z_{5}^{2}$ of which $\operatorname{Prob}\left(L S B\left(\Delta s^{2}\right)=1\right)$ can not be approximated by Remark1 as shown in Section 4. the attack exhaustively searches the remaining key bits.

In this attack, it is possible that the expected time of individual attacks are larger than exhaustively search directly for some key-dependent subsets, which means

$$
2 n+\beta \cdot 2^{8} \geq 2^{8}
$$

Under this condition, the attack also uses exhaustive key search to determine the remaining eight key bits to make sure the time needed not exceed exhaustive search. 
This attack also choose $\alpha \leq 0.01$ to ensure that the attack successes with $99 \%$ probability. In this case, the total expected time complexity is $2^{125.5}$ encryptions.

Our experiment shows that the attack needs at most 75 pairs of encryptions for one test. We ask for $2^{17}$ encryptions which can provide $2^{16}$ pairs of encryptions, which is sufficient for the test. This data complexity $\left(2^{17}\right)$ is the least out of all the known attacks on the 5-round IDEA starting from the first round.

In the second attack, we try to obtain the plaintext pairs satisfying Proposition 1 before the second round. In order to determine $\operatorname{LSB}\left(\Delta s^{3}\right)$, we need to know the least significant bits of $\Delta s^{1}, \Delta s^{2}, \Delta s^{4}$ and $\Delta s^{5}$. Hence, the subkeys we need to know are $Z_{1}^{1}, Z_{2}^{1}, Z_{3}^{1}, Z_{4}^{1}, Z_{5}^{1}, Z_{6}^{1}, Z_{4}^{2}, Z_{5}^{3} . Z_{5}^{4}, Z_{1}^{5}, Z_{2}^{5}, Z_{5}^{5}$ and $Z_{6}^{5}$. These 13 subkeys only cover 107 bits of key $Z(0-106)$. For every guessed 107 key bits, we use similar technique as before. The expected time complexity is $2^{115.3}$, which is the least time complexity out of all the known attacks on the 5-round IDEA starting from the first round.

Because it is not possible to predict the plaintext pairs which produces the intermediate pairs satisfying Proposition 1 before the second round, the encryptions of all the $2^{64}$ plaintexts are required.

\section{Conclusions}

In this paper, we formalized a scheme of identifying the actual key using the key-dependent distribution, called key-dependent attack. How to minimize the time complexity of the key-dependent attack was also discussed. With the keydependent attack, we could improve known cryptanalysis results and obtain more powerful attacks. We presented two key-dependent attacks on IDEA. Our attack on 5.5-round and 6-round variant of IDEA has the least time and data complexities compared with the previous attacks.

We only implemented a tentative exploration of the key-dependent distribution. How to make full use of the key-dependent distribution, especially how to use the key-dependent distribution to improve existing attacks, is worth further studying.

The attack on IDEA makes use of the relation between XOR, modular addition and modular multiplication. We believe that the operation XOR and modular multiplication have more properties that can be explored further [10]. Similar relations among other operations are also valuable to research. The way of making full use of the Biryukov-Demirci Equation to improve attacks on IDEA is also interesting.

\section{References}

1. Ayaz, E.S., Selçuk, A.A.: Improved DST Cryptanalysis of IDEA. In: Biham, E., Youssef, A.M. (eds.) SAC 2006. LNCS, vol. 4356, pp. 1-14. Springer, Heidelberg (2007)

2. Ben-Aroya, I., Biham, E.: Differential Cryptanalysis of Lucifer. J. Cryptology 9(1), 21-34 (1996) 
3. Biham, E., Biryukov, A., Shamir, A.: Miss in the Middle Attacks on IDEA and Khufu. In: Knudsen, L.R. (ed.) FSE 1999. LNCS, vol. 1636, pp. 124-138. Springer, Heidelberg (1999)

4. Biham, E., Dunkelman, O., Keller, N.: Related-Key Boomerang and Rectangle Attacks. In: Cramer, R. (ed.) EUROCRYPT 2005. LNCS, vol. 3494, pp. 507-525. Springer, Heidelberg (2005)

5. Biham, E., Dunkelman, O., Keller, N.: New Cryptanalytic Results on IDEA. In: Lai, X., Chen, K. (eds.) ASIACRYPT 2006. LNCS, vol. 4284, pp. 412-427. Springer, Heidelberg (2006)

6. Biham, E., Dunkelman, O., Keller, N.: A New Attack on 6-Round IDEA. In: Biryukov, A. (ed.) FSE 2007. LNCS, vol. 4593, pp. 211-224. Springer, Heidelberg (2007)

7. Biham, E., Shamir, A.: Differential Cryptanalysis of DES-like Cryptosystems. In: Menezes, A., Vanstone, S.A. (eds.) CRYPTO 1990. LNCS, vol. 537, pp. 2-21. Springer, Heidelberg (1991)

8. Biryukov, A., Nakahara Jr., J., Preneel, B., Vandewalle, J.: New Weak-Key Classes of IDEA. In: Deng, R.H., Qing, S., Bao, F., Zhou, J. (eds.) ICICS 2002. LNCS, vol. 2513, pp. 315-326. Springer, Heidelberg (2002)

9. Borst, J., Knudsen, L.R., Rijmen, V.: Two Attacks on Reduced IDEA. In: Fumy, W. (ed.) EUROCRYPT 1997. LNCS, vol. 1233, pp. 1-13. Springer, Heidelberg (1997)

10. Contini, S., Rivest, R.L., Robshaw, M.J.B., Yin, Y.L.: Improved Analysis of Some Simplified Variants of RC6. In: Knudsen, L.R. (ed.) FSE 1999. LNCS, vol. 1636, pp. 1-15. Springer, Heidelberg (1999)

11. Daemen, J., Govaerts, R., Vandewalle, J.: Weak keys for IDEA. In: Stinson, D.R. (ed.) CRYPTO 1993. LNCS, vol. 773, pp. 224-231. Springer, Heidelberg (1994)

12. Demirci, H.: Square-like Attacks on Reduced Rounds of IDEA. In: Nyberg, K., Heys, H.M. (eds.) SAC 2002. LNCS, vol. 2595, pp. 147-159. Springer, Heidelberg (2003)

13. Demirci, H., Selçuk, A.A., Türe, E.: A New Meet-in-the-Middle Attack on the IDEA Block Cipher. In: Matsui, M., Zuccherato, R.J. (eds.) SAC 2003. LNCS, vol. 3006, pp. 117-129. Springer, Heidelberg (2004)

14. Hawkes, P.: Differential-Linear Weak Key Classes of IDEA. In: Nyberg, K. (ed.) EUROCRYPT 1998. LNCS, vol. 1403, pp. 112-126. Springer, Heidelberg (1998)

15. Hawkes, P., O'Connor, L.: On Applying Linear Cryptanalysis to IDEA. In: Kim, K.-c., Matsumoto, T. (eds.) ASIACRYPT 1996. LNCS, vol. 1163, pp. 105-115. Springer, Heidelberg (1996)

16. Nakahara Jr., J., Preneel, B., Vandewalle, J.: The Biryukov-Demirci Attack on Reduced-Round Versions of IDEA and MESH Ciphers. In: Wang, H., Pieprzyk, J., Varadharajan, V. (eds.) ACISP 2004. LNCS, vol. 3108, pp. 98-109. Springer, Heidelberg (2004)

17. Junod, P.: On the Optimality of Linear, Differential, and Sequential Distinguishers. In: Biham, E. (ed.) EUROCRYPT 2003. LNCS, vol. 2656, pp. 17-32. Springer, Heidelberg (2003)

18. Junod, P.: New Attacks Against Reduced-Round Versions of IDEA. In: Gilbert, H., Handschuh, H. (eds.) FSE 2005. LNCS, vol. 3557, pp. 384-397. Springer, Heidelberg (2005)

19. Kelsey, J., Schneier, B., Wagner, D.: Key-Schedule Cryptoanalysis of IDEA, GDES, GOST, SAFER, and Triple-DES. In: Koblitz, N. (ed.) CRYPTO 1996. LNCS, vol. 1109, pp. 237-251. Springer, Heidelberg (1996) 
20. Knudsen, L.R., Rijmen, V.: On the Decorrelated Fast Cipher (DFC) and Its Theory. In: Knudsen, L.R. (ed.) FSE 1999. LNCS, vol. 1636, pp. 81-94. Springer, Heidelberg (1999)

21. Lai, X.: On the Design and Security of Block Ciphers. ETH Series in Information Processing. Harturg-Gorre Verlag, Konstanz (1992)

22. Lai, X., Massey, J.L.: A Proposal for a New Block Encryption Standard. In: Damgård, I.B. (ed.) EUROCRYPT 1990. LNCS, vol. 473, pp. 389-404. Springer, Heidelberg (1991)

23. Matsui, M.: Linear Cryptoanalysis Method for DES Cipher. In: Helleseth, T. (ed.) EUROCRYPT 1993. LNCS, vol. 765, pp. 386-397. Springer, Heidelberg (1994)

24. Meier, W.: On the Security of the IDEA Block Cipher. In: Helleseth, T. (ed.) EUROCRYPT 1993. LNCS, vol. 765, pp. 371-385. Springer, Heidelberg (1994)

25. Raddum, H.: Cryptanalysis of IDEA-X/2. In: Johansson, T. (ed.) FSE 2003. LNCS, vol. 2887, pp. 1-8. Springer, Heidelberg (2003) 